http://jmscr.igmpublication.org/home/ ISSN (e)-2347-176x ISSN (p) 2455-0450

crossref DOI: https://dx.doi.org/10.18535/jmscr/v9i11.30

\title{
Allopurinol Induced Toxic Epidermal Necrolysis- A Case Report
}

\author{
Authors \\ Dr Jatin Kumar Jain (DNB Medicine Resident), Dr Sunil Rana (Senior Consultant \\ Internal Medicine), Dr Sumit Dhamu, Dr Nisarg Patel \\ Department of General Medicine Sarvodaya Hospital and Research Centre, Faridabad, Haryana
}

\begin{abstract}
Toxic Epidermal Necrolysis (TEN) is as a rare and a life threatening mucocutaneous condition. Drugs are the leading reported cause of TEN, with the risk of a hypersensitivity reaction mainly in the first few weeks of the drug ingestion. There is strong association between TEN and several drugs, including anti-infectives Sulfonamides, allopurinol, carbamazepine, phenobarbital, nevirapine, lamotrigine, phenytoin, and oxicam-non steroidal anti-inflammatory drugs (NSAIDs).TEN is characterized by widespread keratinocyte apoptosis and sloughing of the skin, erosion of the mucous membranes, painful blistering, and severe systemic disturbance. It involves $>30 \%$ of total body surface area.

We report a case of 38year old female who developed Toxic epidermal necrolysis after consumption of Allopurinol for joint pain and was being evaluated prognostically with the SCORTEN score.
\end{abstract}

\section{Introduction}

TEN, a life threatening and a rare Mucocutaneous disorder, most commonly triggered by medications, characterized by extensive necrosis and detachment of the epidermis. ${ }^{[1]}$ Mucous membranes are affected in over 90 percent of patients, usually at two or more distinct sites (ocular, oral, and genital).

TEN can occur in patients of any age. It is more common in women than in men, with a male to female ratio of approximately $1: 2^{[2]}$ The overall mortality rate among patients with Steven Johnson Syndrome (SJS)/Toxic Epidermal necrolysis (TEN) is approximately 30 percent, ranging from approximately 10 percent for SJS to up to 50 percent for TEN. Mortality continues to increase up to one year after disease onset.
Medications are the leading cause of toxic epidermal necrolysis (TEN) in both adults and pediatric population. The risk of TEN is generally limited to the first eight weeks of treatment. The most common drug implicated for TEN are Allopurinol, Aromatic antiepileptic drugs and lamotrigine, Antibacterial sulfonamides (including sulfasalazine), Nevirapine and Oxicam nonsteroidal anti-inflammatory drugs (NSAIDs). Mycoplasma pneumoniae infection - Infections, including Mycoplasma pneumoniae infection, are the next most common trigger of SJS/TEN, particularly in children. ${ }^{[3]}$ In over one-third of SJS/TEN cases, no cause can be identified. Rarely reported causes of TEN include vaccinations, systemic diseases, external chemical exposure, food, contrast medium and herbal medicines. ${ }^{[4]}$ 
Risk Factors of TEN includes, HIV Infection, Malignancy and genetic factors. HIV infected patients have been reported to have an increased risk of drug reactions and a 100-fold higher risk of SJS/TEN than the normal population ${ }^{[5]}$. Patients with any active malignancy (high risk with hematologic cancers) have an increased risk of SJS/TEN ${ }^{[6]}$. Several HLA haplotypes have been implicated in drug-specific susceptibility in certain ethnic groups $^{[7]}$,

\begin{tabular}{|l|c|}
\hline $\begin{array}{l}\text { Drugs increasing risk of } \\
\text { TEN }\end{array}$ & HLA Type \\
\hline Allopurinol & HLA-B*58:01 \\
\hline Sulfonamides & HLA-A29, HLA-B12, \\
& HLA-DR7 \\
\hline NSAIDS & HLA-A2, HLA-B12 \\
\hline Carbamazepine & HLA-B*15:02 \\
\hline
\end{tabular}

The pathologic mechanisms that induce skin damage in Toxic epidermal necrolysis (TEN) is incompletely understood. The few implicated mechanisms for pathogenesis of TEN are:

1) Cell-mediated cytotoxic reaction against keratinocytes leading to massive apoptosis.[90]

2) Drugs stimulating the immune system by directly binding to the major histocompatibility complex (MHC) class I and the T cell receptor.

3) Drugs directly bind to binding to HLA class I peptide pouch and alter the repertoire of peptides recognized as foreign and make the HLA drug complex recognized as foreign.

Clinical presentation of TEN starts with fever, often exceeding $39^{\circ} \mathrm{C}\left(102.2^{\circ} \mathrm{F}\right)$, and influenzalike symptoms preceded by the development of mucocutaneous lesions (ill-defined, coalescing, erythematous macules with purpuric centers while some may present with diffuse erythema). Lesions start on the face and thorax before spreading to other areas and are symmetrically distributed. Nikolsky sign and Asboe-Hansen sign or "bulla spread sign" may also be present.

Mucosal involvement occurs in approximately 90 percent of cases of TEN. Re-epithelialization may begin after several days and typically requires two to four weeks.

Laboratory abnormalities - Hematologic abnormalities, particularly anemia and lymphopenia, are common in TEN [106]. Eosinophilia is unusual; neutropenia is present in approximatelyone-third of patients and is correlated with a poor prognosis. The diagnosis of TEN is based upon clinical and histologic findings in a patient with ahistory of antecedent drug exposure or febrile illness.

Complications include infections which results into Sepsis and septic shock, most often caused by Staphylococcus aureus and Pseudomonas aeruginosa and are the main causes of death in these patients.

\section{Case}

A 38 year old female presented with c/o fever since 3 days, widespread erythematous vesicular skin rash since 2 days, Oral mucosal bleed Vaginal bleed, conjunctivitis since 1 day. She had $\mathrm{h} / \mathrm{o}$ taking allopurinol and paracetamol tablets for joint pain since, 1 week.

On physical examination revealed temp of $102 \mathrm{~F}$ with pulse rate of $112 / \mathrm{min}$

Skin rashes were initially erythematosus and maculopapular and extremely itchy followed by painful blister formation (figure 1 and 2), and also affecting mucous membrane of Oral cavity, anal canal, and vaginal area. Bleeding from the oral and vaginal mucosa was seen, Nikolsky sign was positive.

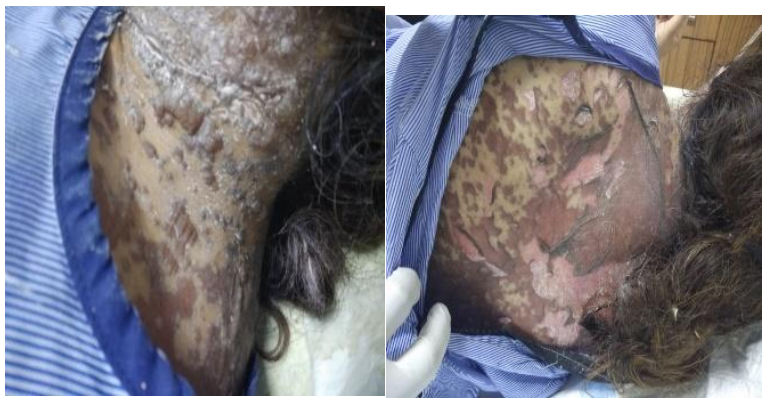

Figure 1 and 2

Laboratory Findings were normal except high Eosinophil counts. 
SCORTEN score was applied for the prognosis of the patient. (table 2$)^{[8]}$

\section{Table 2}

\begin{tabular}{|l|c|}
\hline Prognostic factors & Score \\
\hline Age $>40$ years & 1 \\
\hline Skin involvement $>10 \%$ BSA & 1 \\
\hline Malignancy & 1 \\
\hline Tachycardia $>120 / \mathrm{min}$ & 1 \\
\hline Blood Glucose $>14 \mathrm{mmol} / 1$ & 1 \\
\hline Serum Bicarbonate $<20 \mathrm{mmol} / 1$ & 1 \\
\hline Serum Urea $>10 \mathrm{mmol} / 1$ & 1 \\
\hline
\end{tabular}

Score 0-1 -Mortality 3\%

Score 2- Mortality 12\%

Score 3-Mortality 35\%

Score 4-Mortality 58\%

Score $>=5$ Mortality $90 \%$

\section{SCORTEN SCORE in this case was 1 so prognosis was excellent.}

Patient was treated with IV corticosteroids, IV antibiotics, Oral and IV Antihistamines, Topical Corticosteroids, and other supportive therapy. The lesions gradually diminished and healed up in 15 days and resolved with hypopigmented and hyperpigmented patches.

\section{Discussion}

TEN is a life-threatening immune-mediated drug reaction associated with high morbidity and mortality rates. It is clinically characterized by sudden onset of fever, systemic toxicity, a rapidly progressing erythematous, and purpuric rash with mucosal involvement is also common. Epidermal detachment is more than $30 \%$ BSA in TEN.

The pathogenesis of TEN is not completely understood, but several mechanisms have been implicated like immunologic mechanisms leading to keratinocyte apoptosis, such as, cytotoxic T-cell and natural killer cell damage by perforin/ granzyme $\mathrm{B} /$ granulysin and tumor necrosis factoralpha, in addition to genetic predisposition.

The offending drug should be immediately stopped and therapy is primarily aimed at supportive care with complementary adjuvant therapies like systemic corticosteroids, IVIG, immunosuppressants such as cyclosporine, cyclophosphamide, and thalidomide, and plasmapheresis.

The early application of the SCORTEN score gives an early indication of the prognosis.

\section{References}

1. Stern RS, Divito SJ. Stevens-Johnson Syndrome and Toxic Epidermal Necrolysis: Associations, Outcomes, and Pathobiology-Thirty Years of Progress but Still Much to Be Done. J Invest Dermatol 2017; 137:1004.

2. Sekula P, Dunant A, Mockenhaupt M, et al. Comprehensive survival analysis of a cohort of patients with Stevens-Johnson syndrome and toxic epidermal necrolysis. J Invest Dermatol 2013; 133:1197.

3. Ferrandiz-Pulido C, Garcia-Patos V. A review of causes of Stevens-Johnson syndrome and toxic epidermal necrolysis in children. Arch Dis Child 2013; 98:998.

4. Roujeau JC. Stevens-Johnson syndrome and toxic epidermal necrolysis are severity variants of the same disease which differs from erythema multiforme. J Dermatol 1997; 24:726.

5. Strom BL, Carson JL, Halpern AC, et al. Using a claims database to investigate drug-induced Stevens-Johnson syndrome. Stat Med 1991; 10:565.

6. Rosen AC, Balagula Y, Raisch DW, et al. Life-threatening dermatologic adverse events in oncology. Anticancer Drugs 2014; 25:225.

7. Tangamornsuksan W, Chaiyakunapruk N, Somkrua R, et al. Relationship between the HLAB* 1502 allele and carbamazepine-induced Stevens-Johnson syndrome and toxic epidermal necrolysis: a systematic review and meta-analysis. JAMA Dermatol 2013; 149:1025.

8. Guégan $\mathrm{S}, \quad$ Bastuji-Garin $\mathrm{S}$, Poszepczynska-Guigné E, et al. Performance of the SCORTEN during the 
first five days of hospitalization to predict the prognosis of epidermal necrolysis. $\mathbf{J}$

Invest Dermatol 2006;126:272. 